\title{
Light $D$ wave meson spectrum in a relativistic harmonic model with instanton induced interaction
}

\author{
Antony Prakash Monteiro, Kanti Basavarajappa Vijaya Kumar*
}

Department of Physics, Mangalore University, Mangalagangothri, Mangalore, India; *Corresponding author: kbvijaya kumar@, yahoo.com

Received 20 July 2010; revised 25 August 2010; accepted 28 August 2010.

\begin{abstract}
The mass spectrum of the $D$ wave mesons is considered in the frame work of relativistic harmonic model (RHM). The full Hamiltonian used in the investigation has the Lorentz scalar plus a vector harmonic-oscillator potential, the confined-one-gluon-exchange potential (COGEP) and the instanton-induced quark-antiquark interaction (III). A good agreement between calculated $D$ wave meson masses with experimental $D$ wave meson masses is obtained. The respective role of III and COGEP in the D wave meson spectrum is discussed.
\end{abstract}

Keywords: Quark Model; Confined-One-GluonExchange Potential; Instanton Induced Interaction; D Wave Meson Spectra

\section{INTRODUCTION}

The Quantum Chromodynamics (QCD), the theory of strong interactions, is not exactly solvable in the non-perturbative regime which is required to obtain the physical properties of the hadrons. Hence various approximation methods like lattice gauge theories are employed to solve QCD in the non-perturbative regime. In the constituent quark model, conventional mesons are bound states of a spin $1 / 2$ quark and spin $1 / 2$ antiquark bound by a phenomenological potential. The phenomenological models developed to explain the observed properties of mesons are either non-relativistic quark models (NRQM) with suitably chosen potential or relativistic models where the interaction is treated perturbatively [1-3]. In most of the works that use NRQM, it is assumed that the quark interaction is dominated by a linear or quadratic confinement potential and is supplemented by a short range potential stemming from the one-gluon exchange mechanism. The Hamiltonian of these quark models usually contains three main ingredi- ents: the kinetic energy, the confinement potential and a hyperfine interaction term, which has often been taken as an effective one-gluon-exchange potential (OGEP) [4]. Other types of hyperfine interaction like InstantonInduced Interaction (III) deduced by a non-relativistic reduction of the 't Hooft interaction [5-12] have also been introduced in the literature.

The success of the NRQM in describing the hadron spectrum is somewhat paradoxical, as light quarks should in principle not obey a non-relativistic dynamics. This paradox has been avoided in many works based on the constituent quark models by using for the kinetic energy term of the Hamiltonian a semi-relativistic or relativistic expression [9-12]. Even in the existing relativistic models though the effect of confinement of quarks has been taken into account, the effect of confinement of gluons has not been taken into account [13-15]. Therefore in our present work, we have investigated the effect of exchange of confinement of gluons on the masses of light $\mathrm{D}$ wave mesons and their radially excited states in the frame work of RHM with III $[6,11-12,16]$. The essential new ingredient in our investigation of the light $\mathrm{D}$ wave mesonic states is to take into account the confinement of gluons in addition to the confinement of quarks. In the existing quark models, Fermi-Breit interaction which gives rise to $\pi-\rho$ and $N-\Delta$ splitting is treated as perturbation. The OGEP being attractive for $\pi$, and for a nucleon a naïve perturbative treatment of one gluon hyperfine interaction is incorrect and hence one obtains a high value for the pion mass. This leads to further renormalization of strength of interaction for a better fit. Also, the most prominent flaw of NRQM is the neglect of relativistic effects and gluon dynamics. In our present work, for the confinement of quarks we are making use of the RHM which has been successful in explaining the properties of light hadrons. For the confinement of gluons, we have made use of the current confinement model (CCM) which was developed in the spirit of the RHM [13-15]. The CCM has been quite successful in describing the glue-ball spectra. The confined gluon 
propagators (CGP) are derived in CCM. Using CGP we have obtained confined one gluon exchange potential (COGEP). The full Hamiltonian used in the investigation has Lorentz scalar plus a vector harmonic-oscillator potential, in addition to two-body COGEP and III. In our present work we are extending the model to study $\mathrm{D}$ wave light meson spectra. In our present work, the total mass of the meson is obtained by calculating the energy eigen values of the Hamiltonian in the harmonic oscillator basis spanned over a space extending upto the radial quantum number $\mathrm{n}_{\max }=4$. The masses of $\mathrm{D}$ wave mesons are obtained after diagonalising for various values of $n_{\max }$.

In Section 2, we review the RHM and CCM models and give a brief description of the CGP, COGEP and III. We also discuss the parameters involved in our model. The results of the calculations are presented in Section 3. Conclusions are given in Section 4.

\section{THE MODEL}

In RHM, quarks in a hadron are confined through the action of a Lorentz scalar plus a vector harmonic-oscillator potential [13-15].

$$
V_{\text {conf }}(\vec{r})=\frac{1}{2}\left(1+\gamma_{0}\right) A^{2} r^{2}+M
$$

where $\gamma_{0}$ is the Dirac matrix:

$$
\gamma_{0}=\left(\begin{array}{cc}
1 & 0 \\
0 & -1
\end{array}\right)
$$

$M$ is the quark mass and $A^{2}$ is the confinement strength. They have a different value for each quark flavour. In RHM, the confined single quark wave function $(\psi)$ is given by:

$$
\psi=N\left(\begin{array}{c}
\phi \\
\frac{\boldsymbol{\sigma} \cdot \mathbf{P}}{E+M} \phi
\end{array}\right)
$$

with the normalization

$$
N=\sqrt{\frac{2(E+M)}{3 E+M}} ;
$$

where $\mathrm{E}$ is an eigenvalue of the single particle Dirac equation with the interaction potential given in (1). The lower component is eliminated by performing the similarity transformation,

$$
U \psi=\phi
$$

where $\mathrm{U}$ is given by,

$$
\frac{1}{N\left[1+\frac{\mathbf{P}^{2}}{(E+M)^{2}}\right]}\left(\begin{array}{cc}
\mathbf{1} & \frac{\boldsymbol{\sigma} \cdot \mathbf{P}}{E+M} \\
-\frac{\boldsymbol{\sigma} \cdot \mathbf{P}}{E+M} & \mathbf{1}
\end{array}\right)
$$

formation operator. With this transformation, the upper component $\phi$ satisfies the harmonic oscillator wave equation.

$$
\left[\frac{\mathbf{P}^{2}}{E+M}+A^{2} r^{2}\right] \phi=(E-M) \phi
$$

which is like the three dimensional harmonic oscillator equation with an energy-dependent parameter $\Omega_{n}^{2}$ :

$$
\Omega_{n}=A\left(E_{n}+M\right)^{1 / 2}
$$

The eigenvalue of (7) is given by,

$$
E_{n}^{2}=M^{2}+(2 n+1) \Omega_{n}^{2} .
$$

Note that Eq.7 can also be derived by eliminating the lower component of the wave function using the Foldy-Wouthuysen transformation as it has been done in [13].

Adding the individual contributions of the quarks, we obtain the total mass of the hadron. The spurious centre of mass $(\mathrm{CM})$ is corrected by using intrinsic operators for the $\sum_{i} r_{i}^{2}$ and $\sum_{i} \nabla_{i}^{2}$ terms appearing in the Hamiltonian. This amounts to just subtracting the CM motion zero point contribution from the $E^{2}$ expression. It should be noted that this method is exact for the 0S-state quarks as the $\mathrm{CM}$ motion is also in the $0 \mathrm{~S}$ state.

The two body quark-antiquark potential is the sum of COGEP and III potential.

$$
V_{q}\left(\vec{r}_{i j}\right)=V_{\text {COGEP }}\left(\vec{r}_{i j}\right)+V_{I I I}\left(\vec{r}_{i j}\right)
$$

COGEP is obtained from the scattering amplitude [3,13-15],

$$
M_{f i}=\frac{g_{s}^{2}}{4 \pi} \bar{\psi}_{i} \gamma^{\mu} \frac{\lambda_{i}^{a}}{2} \psi_{i} D_{\mu \nu}^{a b}(q) \bar{\psi}_{j} \gamma^{\nu} \frac{\lambda_{j}^{b}}{2} \psi_{j},
$$

where, $\bar{\psi}=\psi^{+} \gamma_{0}, \psi_{i / j}$ are the wave functions of the quarks in the RHM, $D_{\mu \nu}^{a b}=\partial_{a b} D_{\mu \nu}$ are the CCM gluon propagators in momentum representation, $g_{s}^{2} / 4 \pi \quad\left(=\alpha_{s}\right)$ is the quark-gluon coupling constant and $\lambda_{i}$ is the color $S U(3)_{c}$ generator of the $i^{\text {th }}$ quark. The details can be found in references $[3,13]$. Below, we list the expressions for the central, tensor and spin-orbit part of the COGEP.

The central part of COGEP is [3,13-15],

$$
\begin{aligned}
& V_{\text {COGEP }}^{\text {cent }}\left(\vec{r}_{i j}\right)=\frac{\alpha_{s} N^{4}}{4} \lambda_{i} \cdot \lambda_{j} \\
& {\left[D_{0}\left(\vec{r}_{i j}\right)+\frac{1}{(E+M)^{2}}\left[4 \pi \delta^{3}\left(\vec{r}_{i j}\right)-c^{4} r^{2} D_{1}\left(\vec{r}_{i j}\right)\right]\left[1-2 / 3 \boldsymbol{\sigma}_{i} \cdot \boldsymbol{\sigma}_{j}\right]\right]}
\end{aligned}
$$

To calculate the matrix elements (ME) of COGEP, we have fitted the exact expressions of $D_{0}(\vec{r})$ and $D_{1}(\vec{r})$ by Gaussian functions. It is to be noted that the $D_{0}(\vec{r})$ and $D_{1}(\vec{r})$ are different from the usual Coulombic propagators. However, in the asymptotic limit $(\vec{r} \rightarrow 0)$ they are similar to Columbic propagators and in the infrared limit $(\vec{r} \rightarrow \infty)$ they fall like Gaussian. In the 
above expression the $\mathrm{c}\left(\mathrm{fm}^{-1}\right)$ gives the range of propagation of gluons and is fitted in CCM $[13,14]$ to obtain the glue ball spectra. The $D_{0}(\vec{r})$ and $D_{1}(\vec{r})$ are given by,

$D_{0}(\vec{r})=\left(\frac{\alpha_{1}}{r}+\alpha_{2}\right) \exp \left[\frac{-r^{2} c_{0}^{2}}{2}\right] ; D_{1}(\vec{r})=\frac{\gamma}{r} \exp$ $\left[\frac{-r^{2} c_{0}^{2}}{2}\right]$

where $\alpha_{1}=1.035994, \alpha_{2}=2.016150 \mathrm{fm}^{-1}, c_{0}=$ $(3.001453)^{1 / 2} \mathrm{fm}^{-1,} \gamma=0.8639336$.

And $c_{2}=(4.367436)^{1 / 2} \mathrm{fm}^{-1}$.

Tensor part of COGEP is,

$$
\begin{gathered}
V_{C O G E P}^{T E N}\left(\vec{r}_{i j}\right)=-\frac{\alpha_{s}}{4} \lambda_{i} \cdot \lambda_{j} \frac{\mathrm{N}^{4}}{(E+M)^{2}} \\
\left(\frac{D_{1}^{\prime \prime}\left(\vec{r}_{i j}\right)}{3}-\frac{D_{1}^{\prime}\left(\vec{r}_{i j}\right)}{3 r}\right) \hat{\mathrm{S}}_{\mathrm{ij}}
\end{gathered}
$$

where $\hat{\mathrm{S}}_{i j}=\left[3\left(\boldsymbol{\sigma}_{i} \cdot \hat{r}\right)\left(\boldsymbol{\sigma}_{j} \cdot \hat{r}\right)-\boldsymbol{\sigma}_{i} \cdot \boldsymbol{\sigma}_{j}\right]$

where $\hat{\mathbf{r}}=\hat{\mathbf{r}}_{i}-\hat{\mathbf{r}}_{j}$ is the unit vector in the direction of $\vec{r}$. In the above expression primes and double primes corresponds to first and second derivatives of $D_{1}(\vec{r})$. The derivatives of $D_{1}(\vec{r})$ were fitted to Gaussian functions.

$$
\begin{aligned}
& D_{1}^{\prime}\left(\vec{r}_{i j}\right)=\frac{1}{r} \varepsilon \exp \left[\frac{-r^{2} c_{3}^{2}}{2}\right]-\frac{1}{r^{2}} \gamma \exp \left[\frac{-r^{2} c_{2}^{2}}{2}\right] \\
& D_{1}^{\prime \prime}\left(\vec{r}_{i j}\right)= \\
& D_{1}^{\prime \prime}\left(\vec{r}_{i j}\right) \frac{2}{r^{3}} \gamma \exp \left[\frac{-r^{2} c_{2}^{2}}{2}\right]-\frac{2}{r^{2}} \varepsilon \exp \left[\frac{-r^{2} c_{3}^{2}}{2}\right]+ \\
& \frac{1}{r} \kappa r^{2} \exp \left[-\frac{r^{2} c_{4}^{2}}{2}\right] \\
& V_{C O G E P}^{L S}\left(\vec{r}_{i j}\right)=-\frac{\alpha_{s}}{4} \lambda_{i} \cdot \lambda_{j} \frac{\mathrm{N}^{4}}{(E+M)^{2}} \frac{1}{2 r} \\
& \left(\left[\vec{r}_{i j} \times\left(\vec{p}_{i}-\vec{p}_{j}\right) \cdot\left(\boldsymbol{\sigma}_{i}+\boldsymbol{\sigma}_{j}\right)\right]\left[D_{0}^{\prime}\left(\vec{r}_{i j}\right)+2 D_{1}^{\prime}\left(\vec{r}_{i j}\right)\right]\right) \\
& D_{0}^{\prime}\left(\vec{r}_{i j}\right)= \\
& \frac{1}{r}\left[\beta_{1}+r \beta_{2}\right] \exp \left[\frac{-r^{2} c_{1}^{2}}{2}\right]-\frac{1}{r^{2}}\left[\alpha_{1}+r \alpha_{2}\right] \exp \left[\frac{-r^{2} c_{0}^{2}}{2}\right] \\
& 2 D_{1}^{\prime}\left(\vec{r}_{i j}\right)=2\left(\frac{1}{r} \varepsilon \exp \left[\frac{-r^{2} c_{3}^{2}}{2}\right]-\frac{1}{r^{2}} \gamma \exp \left[\frac{-r^{2} c_{2}^{2}}{2}\right]\right)
\end{aligned}
$$

where $\beta_{1}=2.680358 \mathrm{fm}^{-1}, \quad \beta_{2}=-7.598860 \mathrm{fm}^{-2}$ and $c_{1}=$ $(2.373588)^{1 / 2}$.

It should be noted that in the limit $\mathrm{c} \rightarrow 0$, the central, tensor and spin-orbit part of the COGEP goes over to the corresponding potentials of the OGEP [3].

The central part of III potential is given by $[8-12,16]$,

$$
V_{I I I}=\left\{\begin{array}{c}
-8 g \delta\left(r_{i j}\right) \delta_{S, 0} \delta_{L, 0}, \text { for } I=1, \\
-8 g^{\prime} \delta\left(r_{i j}\right) \delta_{S, 0} \delta_{L, 0}, \text { for } I=1 / 2, \\
8\left(\begin{array}{cc}
g & \sqrt{2} g^{\prime} \\
\sqrt{2} g^{\prime} & 0
\end{array}\right) \delta\left(r_{i j}\right) \delta_{S, 0} \delta_{L, 0}, \text { for } I=0
\end{array}\right.
$$

The symbols S, L and I are respectively the spin, the relative angular momentum and the iso-spin of the system. The $g$ and $g^{\prime}$ are the coupling constants of the interaction. The Dirac delta-function appearing has been regularized and replaced by a Gaussian- like function:

$$
\delta_{i j} \rightarrow \frac{1}{(\Lambda \sqrt{\pi})^{3}} \exp \left[-\frac{r_{i j}^{2}}{\Lambda^{2}}\right]
$$

where $\Lambda$ is the size parameter.

The non-central part of III has contributions from both spin- orbit and tensor terms. The spin-orbit contribution comes from relativistic corrections to the central potential of III. It is given by [8-12],

$$
V_{I I I}^{S O}\left(\vec{r}_{i j}\right)=V_{L S}\left(\vec{r}_{i j}\right) \vec{L} \cdot \vec{S}+V_{L \Delta}\left(\vec{r}_{i j}\right) \vec{L} \cdot \vec{\Delta}
$$

The first term in Eq.17 is the traditional symmetric spin-orbit term proportional to the operator $\vec{L} \cdot \vec{S}$. The other term is the anti-symmetric spin-orbit term proportional to $\vec{L} \cdot \vec{\Delta}$ where $\vec{\Delta}=\frac{1}{2}\left(\vec{\sigma}_{1}-\vec{\sigma}_{2}\right)$. The radial functions of Eq.17 are expressed as [8-12],

$$
\begin{aligned}
& V_{L S}\left(\vec{r}_{i j}\right)=\left(\frac{1}{M_{i}^{2}}+\frac{1}{M_{j}^{2}}\right) \sum_{k=1}^{2} \kappa_{k} \frac{\exp \left(-\mathrm{r}_{\mathrm{ij}}^{2} / \eta_{k}^{2}\right)}{\left(\eta_{k} \sqrt{\pi}\right)^{3}}+ \\
& \left(\frac{1}{M_{i} M_{j}}\right) \sum_{k=3}^{4} \kappa_{k} \frac{\exp \left(-\mathrm{r}_{\mathrm{ij}}^{2} / \eta_{k-2}^{2}\right)}{\left(\eta_{k-2} \sqrt{\pi}\right)^{3}}
\end{aligned}
$$

and

$$
V_{L \Delta}\left(\vec{r}_{\mathrm{ij}}\right)=\left(\frac{1}{M_{i}^{2}}-\frac{1}{M_{j}^{2}}\right) \sum_{k=5}^{6} \kappa_{k} \frac{\exp \left(-\mathrm{r}_{\mathrm{ij}}^{2} / \eta_{k-4}^{2}\right)}{\left(\eta_{k-4} \sqrt{\pi}\right)^{3}}
$$

The term $V_{L S}(\vec{r})$ is responsible for the spitting of the ${ }^{3} L_{J}$ states with $J=L-1, \mathrm{~L}, \mathrm{~L}+1$. With such a term $L$ is still good quantum numbers but $\mathrm{S}$ is not. The term $V_{L \Delta}(\vec{r})$ which couples states ${ }^{1} L_{J=L}$ and ${ }^{3} L_{J=L}$. Due to the mass dependence in Eq.19, it is clear that this term is inoperative when the quarks are identical. In practice the antisymmetric spin obit term is important 
only in the K-sector. The terms $\kappa_{\mathrm{i}}$ and $\eta_{\mathrm{i}}$ are free parameters in the theory [12]. $M_{i}$ corresponds to the mass of the strange quark (s) and $M_{j}$ corresponds to mass of $(\mathrm{u} / \mathrm{d})$ quark. This term accounts for the splitting between $1^{1} \mathrm{D}_{2}$ and $1{ }^{3} \mathrm{D}_{2}$ states in the $\mathrm{K}$ sector.

The tensor interaction of III is [12],

$$
V_{I I I}^{T E N}\left(\vec{r}_{\mathrm{ij}}\right)=\frac{\hat{S}_{i j}}{M_{i} M_{j}} \sum_{k=7}^{8} \kappa_{k} \frac{\exp \left(-\mathrm{r}_{\mathrm{ij}}^{2} / \eta_{k-4}^{2}\right)}{\left(\eta_{k-4} \sqrt{\pi}\right)^{3}}
$$

with the tensor interaction, $\mathrm{L}$ is no longer a good quantum number since this term couples the states ${ }^{3} L_{J=L+1}$ and ${ }^{3}(L+2)_{J=L+1}$.

The parameters of the RHM are the masses of the quarks, $M_{u}=M_{d}$ and $\mathrm{Ms}$, the respective confinement strengths, $\mathrm{A}_{\mathrm{u}}{ }^{2}=\mathrm{A}_{\mathrm{d}}{ }^{2}, \mathrm{~A}_{\mathrm{s}}{ }^{2}$, and the oscillator size parameter $b_{n}\left(=1 / \Omega_{n}\right)$. They have been chosen to reproduce various nucleon properties: the root mean square charge radius, the magnetic moment and the ratio of the axial coupling to the vector coupling [13]. The confinement strength $A_{u, d}$ is fixed by the stability condition for the nucleon mass against the variation of the size parameter $b_{n}$

$$
\frac{\partial}{\partial b_{n}}\langle N|H| N\rangle=0
$$

The parameters associated with the strange quark $M_{s}$ and $\mathrm{A}_{\mathrm{s}}{ }^{2}$ have been fitted in order to reproduce the magnetic moments of the strange baryons, according to the procedure described in [16]. The $\alpha_{s}$ of COGEP is fixed from $S$ wave meson spectroscopy [16]. The value of $\alpha_{s}$ turns out to be 0.2 for $\mathrm{D}$ wave mesons, which is compatible with the perturbative treatment. Among the non central parts of the potential, the hyperfine terms of III has 12 additional strength and size parameters $\kappa$ 's and $\eta$ 's ( in Eqns. 18-20) respectively. We note that the $\kappa$ values can have both positive and negative values [8-12]. The values of the III parameters $\kappa$ 's and $\eta$ 's are fixed from $\mathrm{S}$ and $\mathrm{P}$ wave meson spectroscopy $[12,16]$ and are listed in Table $\mathbf{1}$ and $\mathbf{2}$.

Table 1. The parameters for the D wave mesons.

\begin{tabular}{cc}
\hline $\mathrm{b}$ & $0.62 \mathrm{fm}$ \\
\hline $\mathrm{M}_{\mathrm{u}, \mathrm{d}}$ & $380 \mathrm{MeV}$ \\
$\mathrm{M}_{\mathrm{s}}$ & $560 \mathrm{MeV}$ \\
$\alpha_{s}$ & 0.2 \\
$\eta_{1}$ & $0.2 \mathrm{fm}$ \\
$\eta_{2}$ & $0.29 \mathrm{fm}$ \\
$\eta_{3}$ & $1.4 \mathrm{fm}$ \\
$\eta_{4}$ & $1.3 \mathrm{fm}$ \\
$\kappa_{1}$ & 1.8 \\
$\kappa_{2}$ & 1.7 \\
$\kappa_{3}$ & 1.9 \\
$\kappa_{4}$ & 2.1 \\
$\kappa_{5}$ & -22.0 \\
$\kappa_{6}$ & -24.5 \\
\hline
\end{tabular}

Table 2. Values of $\kappa_{7}$ and $\kappa_{8}$ parameters.

\begin{tabular}{ccc}
\hline Meson & $\kappa_{7}$ & $\kappa 8$ \\
\hline$\omega(1650)$ & 28.0 & 36.0 \\
$\mathrm{~K}^{*}(1680)$ & 49.0 & 52.0 \\
$\mathrm{~K}_{2}(1820)$ & 36.0 & 45.0 \\
$\omega_{3}(1670)$ & -1.6 & -6.5 \\
$\mathrm{~K}^{*}(1780)$ & 1.0 & -2.5 \\
$\phi_{3}(1850)$ & -4.0 & -8.0 \\
\hline
\end{tabular}

\section{RESULTS AND DISCUSSIONS}

To calculate the meson masses, the product of quark-antiquark oscillator wave functions is expressed in terms of oscillator wave functions corresponding to the relative and $\mathrm{CM}$ coordinates. The oscillator quantum number for the $\mathrm{CM}$ wave functions is restricted to $\mathrm{N}_{\mathrm{CM}}=$ 0 . The Hilbert space of relative wave functions is truncated at radial quantum number $\mathrm{n}_{\max }=4$. The Hamiltonian matrix is constructed for each meson separately in the basis states of $\left|N_{C M}=0, L_{C M}=0 ;{ }^{2 S+1} L_{J}\right\rangle$ and diagonalised.

We have obtained the D wave meson spectra using the model described above. The masses of the singlet and triplet $\mathrm{D}$ wave mesons after diagonalisation in harmonic oscillator basis with $\mathrm{n}_{\max }=4$ are listed in Tables 3 and $\mathbf{4}$ respectively. The results show that III potential along with COGEP is necessary to obtain the meson mass spectra. If COGEP is taken as the only source of hyperfine interaction, the value of $\alpha_{s}$ necessary to reproduce the hadrons spectrum is generally much larger than one; this leads to a large spin-orbit interaction, which destroys the overall fit to the spectrum. The inclusion of III diminishes the relative importance of COGEP for the hyperfine splitting. The important role played by III in obtaining the masses of these mesons can be well understood by examining the Table 6. In Table 6, we have given the calculated masses of triplet $\mathrm{D}$ wave mesons

Table 3. Masses of the singlet mesons (in $\mathrm{MeV}$ ).

\begin{tabular}{cccc}
\hline $\mathrm{N}^{2 \mathrm{~S}+1} \mathrm{~L}_{\mathrm{J}}$ & Meson & $\begin{array}{c}\text { Experimental } \\
\text { Mass }\end{array}$ & $\begin{array}{c}\text { Calculated } \\
\text { Mass }\end{array}$ \\
\hline \multirow{2}{*}{$1^{1} \mathrm{D}_{2}$} & $\pi_{2}(1670)$ & $1670 \pm 20$ & 1673.8 \\
& $\mathrm{~K}_{2}(1770)$ & $1773 \pm 8$ & 1768.8 \\
\hline
\end{tabular}

Table 4. Masses of the triplet mesons ( in MeV).

\begin{tabular}{cccc}
\hline $\mathrm{N}^{2 \mathrm{~S}+1} \mathrm{~L}_{\mathrm{J}}$ & Meson & $\begin{array}{c}\text { Experimental } \\
\text { Mass }\end{array}$ & Calculated Mass \\
\hline \multirow{2}{*}{$1^{3} \mathrm{D}_{1}$} & $\omega(1650)$ & $1649 \pm 24$ & 1649.6 \\
$1^{3} \mathrm{D}_{2}$ & $\mathrm{~K}^{*}(1680)$ & $1717 \pm 27$ & 1718.9 \\
& $\mathrm{~K}_{2}(1820)$ & $1816 \pm 13$ & 1818.6 \\
$1^{3} \mathrm{D}_{3}$ & $\mathrm{~K}_{3}(1670)$ & $1667 \pm 4$ & 1667.8 \\
& $\phi_{3}(1850)$ & $1776 \pm 7$ & 1778.3 \\
& & $1854 \pm 7$ & 1855.9 \\
\hline
\end{tabular}


Table 5. The diagonal contributions to the masses of mesons by $\mathrm{V}_{\text {conf }}$, color-electric (CE), color-magnetic (CM), spin-orbit, tensor terms of COGEP and spin-orbit, tensor terms of III (in MeV).

\begin{tabular}{cccccccc}
\hline Meson & $\mathrm{V}_{\text {conf }}$ & $V_{\text {COGEP }}^{C E}$ & $V_{\text {COGEP }}^{C M}$ & $\mathrm{~V}_{\text {COGEP }}^{\text {LS }}$ & $\mathrm{V}_{\text {COGEP }}^{\text {TEN }}$ & $\mathrm{V}_{\text {III }}^{\text {LS }}$ & $\mathrm{V}_{\text {III }}^{\text {TEN }}$ \\
\hline$\pi_{2}(1670)$ & 1675.26 & -2.83 & 1.43 & $\ldots$ & $\ldots$ & $\ldots$ & $\ldots$ \\
$\mathrm{K}_{2}(1770)$ & 1770.79 & -3.13 & 1.22 & $\ldots$ & $\ldots$ & $\ldots$ & $\ldots$ \\
$\omega(1650)$ & 1675.26 & -2.83 & -0.48 & 2.12 & 0.44 & -41.83 & -257.09 \\
$\mathrm{~K}^{*}(1680)$ & 1770.79 & -3.13 & -0.41 & 1.81 & -0.38 & -29.74 & -273.60 \\
$\mathrm{~K}_{2}(1820)$ & 1770.79 & -3.13 & -0.41 & 0.60 & 0.38 & -9.91 & 220.59 \\
$\omega_{3}(1670)$ & 1675.26 & -2.83 & -0.48 & -1.41 & -0.13 & 27.89 & 9.59 \\
$\mathrm{~K}^{*}(1780)$ & 1770.79 & -3.13 & -0.41 & -1.21 & -0.11 & 19.83 & 1.34 \\
$\phi_{3}(1850)$ & 1866.32 & -3.42 & -0.35 & -1.05 & -0.09 & 12.84 & 6.43 \\
\hline
\end{tabular}

Table 6. Masses of triplet mesons ( in MeV) without III.

\begin{tabular}{ccc}
\hline Meson & Experimental Mass & $\begin{array}{c}\text { Calculated Mass } \\
\text { without III }\end{array}$ \\
\hline$\omega(1650)$ & $1649 \pm 24$ & 1676.3 \\
$\mathrm{~K}^{*}(1680)$ & $1717 \pm 27$ & 1770.9 \\
$\mathrm{~K}_{2}(1820)$ & $1816 \pm 13$ & 1768.2 \\
$\omega_{3}(1670)$ & $1667 \pm 4$ & 1670.4 \\
$\mathrm{~K}^{*}(1780)$ & $1776 \pm 7$ & 1765.9 \\
$\phi_{3}(1850)$ & $1854 \pm 7$ & 1861.4 \\
\hline
\end{tabular}

without the inclusion of III potential. The role of III is crucial in explaining the mass differences of $\mathrm{D}$ wave $\mathrm{K}$ mesons. The Table 5 gives the diagonal contributions to the masses of $\mathrm{D}$ wave mesons by the confinement potential, colour-electric $(\mathrm{CE})$, colour-magnetic $(\mathrm{CM})$, spinorbit, tensor terms of COGEP and spin-orbit, tensor terms of III. In case of singlet D wave mesons, tensor and spin-orbit terms of COGEP and III do not contribute to the masses. In case of these singlet mesons the CE part of COGEP is attractive, whereas the CM part of COGEP is repulsive. The dominant contribution to the calculated masses comes from the confinement potential. In case of triplet $\mathrm{D}$ wave mesons the contribution of III potential is very significant. From Table 5, we note that the significant contribution to the masses of $1^{3} \mathrm{D}_{1}$ mesons arises from the tensor term of III which is attractive. The tensor term involves the parameters $\mathrm{k}_{7}$ and $\mathrm{k}_{8}$. It was necessary to tune $\mathrm{k}_{7}$ and $\mathrm{k}_{8}$ parameters to get a reasonably good agreement with the experimental masses. Hence in our model, we have only two free parameters $\mathrm{k}_{7}$ and $\mathrm{k}_{8}$. For $1^{3} \mathrm{D}_{1}$ mesons along with the tensor contribution of III, the spin orbit contribution of III is also significant and is attractive. The contribution of tensor term of III in case of $1^{3} \mathrm{D}_{2}$ is repulsive that significantly increases the value of calculated mass. It is to be noted that the anti-symmetric spin orbit potential of III contributes substantially to the mass difference between the $1^{1} \mathrm{D}_{2}$ and $1^{3} \mathrm{D}_{2}$ mesons in the $\mathrm{K}$ meson sector. The mass difference between $\mathrm{K}^{*}(1680)$ and $\mathrm{K}_{2}(1820)$ mesons is due to the large difference in tensor part of III potential. The tensor III potential is attractive for $\mathrm{K}^{*}(1680)$ but is repulsive for $\mathrm{K}_{2}(1820)$ [17]. In case of $1^{3} \mathrm{D}_{3}$ mesons the contribution due to spin-orbit part of III potential is dominant compared to that of tensor part which is repulsive. From Tables 3 and $\mathbf{4}$, it is clear that the calculated meson masses are in good agreement with the experimental masses [18].

\section{CONCLUSIONS}

We have investigated the effect of the III on the masses of the D wave mesons in the frame work of RHM. We have shown that the computation of the masses using only COGEP is inadequate. The contribution of the III is found to be significant. To obtain the masses of $\mathrm{D}$ wave mesons, $5 \times 5$ Hamiltonian matrix was diagonalised. The contribution from the tensor and spin-orbit part of the III is found to be significant in case of triplet D wave mesons. To obtain the physical masses of the mesons in the $\mathrm{K}$ sector it is necessary to include the anti-symmetric part of III. There is a good agreement between the calculated and experimental masses of $\mathrm{D}$ wave mesons. To enhance the performance of the model, there is a need to make a global chi-square fit of the parameters and also to test different wave functions which would ultimately give better result. Also, the model has to be tested to calculate the leptonic and radiative decay widths. Work in this direction is in progress.

\section{ACKNOWLEDGEMENTS}

One of the authors (APM) is grateful to the DST, India, for granting the JRF. The other author (KBV) acknowledges the DST for funding the project (Sanction No. SR/S2/HEP-14/2006) and the fruitful discussion he had with Y.L. Ma of IHEP, Beijing.

\section{REFERENCES}

[1] Bhaduri, R.K., Cohler, L.E. and Nogami, Y. (1981) A unified potential for mesons and baryons. Nuovo Ci- 
mento A, 65, 376-390.

[2] Godfrey, S. and Isgur, N. (1985) Mesons in a relativised quark model with chromodynamics. Physical Review D, 32, 189-231.

[3] Vinodkumar, P.C., Vijaya Kumar, K.B. and Khadkikar, S.B. (1992) Effect of the confined gluons in quark-quark interaction. Pramana-Journal of Physics, 39, 47-70.

[4] De Rujula, A., Georgi, H. and Glashow, S.L. (1975) Hadron masses in a gauge theory. Physical Review D, 12, 147-162.

[5] 't. Hooft, G. (1976) Computation of the quantum effects due to a four dimensional pseudoparticle. Physical Review $D, \mathbf{1 4}, 3432-3450$.

[6] Vijaya Kumar, K.B., Hanumaiah, B. and Pepin, S. (2004) Meson spectrum in a relativistic harmonic model with instanton- induced interaction. The European Physical Journal A, 19, 247-250.

[7] Blask, W.H., Bohn, U., Huber, M.G., Metsch, B.C. and Petry, H.R. (1990) Hadron spectroscopy with insatanton induced quark forces.Z. Journal of Physics A, 337, 327335.

[8] Brau, F., Semay, C. and Silvestre- Brac, B. (2000) Semiclassical model of light mesons. Physical Review D, 62, 1175011-1175014.

[9] Semay, C. and Silvestre- Brac, B. (1997) Potential models and meson spectra. Nuclear Physics A, 618, 455-482.

[10] Semay, C. and Silvestre-Brac, B. (1999) Determination of quark- antiquark potentials and meson spectra. $\mathrm{Nu}$ - clear Physics A, 647, 72-96.

[11] Bhavyashri, Vijaya Kumar, K.B., Hanumaiah, B., Sarangi, S. and Zhou, S.G. (2005) Meson spectrum in a non relativistic model with instanton-induced interaction. Journal of Physics G: Nuclear and Particle Physics, 31, 981-986.

[12] Bhavyashri, Prakash, A., Ma, Y.L. and Vijaya Kumar, K.B. (2009) $P$ wave meson spectrum in a relativistic model with instanton induced interaction. International Journal of Modern Physics A, 24, 4209-4220.

[13] Khadkikar, S.B. and Gupta, S.K. (1983) Magnetic moments of light baryons in harmonic Model. Physics Letters $B, \mathbf{1 2 4}, 523-526$.

[14] Khadkikar, S.B. and Vijaya Kumar, K.B. (1991) N-N scattering with exchange of confined gluons. Physics Letters B, 254, 320-324.

[15] Vijaya Kumar, K.B. and Khadkikar, S.B. (1998) Spin observables of the $\mathrm{NN}$ interaction in a relativistic harmonic model with confined gluons and mesons. Pramana Journal of Physics, 50, 149-153.

[16] Monteiro, A.P. and Vijaya Kumar, K.B. (2009) Ground state meson spectrum in a relativistic model with instanton induced interaction. Communications in theoretical Physics, 52, 1-6,

[17] Burakovsky, L. and Goldman, T. (1997) On D-wave meson spectroscopy and the $\mathrm{K}^{*}(1410)-\mathrm{K}^{*}(1680)$ problem. Nuclear Physics A, 625, 220-230.

[18] Amsler, C. et al. (2008) Particle Data Group, Physical Letters $B, \mathbf{6 6 7}, 1$ 